\title{
MICROLENSING WITH BINARIES AND PLANETS
}

\author{
H.J. WITT ${ }^{1}$ AND S. MAO ${ }^{2}$ \\ ${ }^{1}$ AIP, An der Sternwarte 16, 14482 Potsdam, Germany \\ ${ }^{2}$ CfA, MS 51, 60 Garden Street, Cambridge, MA 02138, USA
}

\section{Introduction}

The ongoing microlensing experiments have now discovered more than 70 candidate events (Alcock et al. 1993, Bennett et al. 1994, Aubourg et al. 1993, Udalski et al. 1994). These experiments have put important constraints on the dark matter content of the Galactic halo (Alcock et al. 1995a) and yielded many interesting results about Galactic structure (Paczyński et al. 1994, Stanek et al. 1994).

A subclass of these lensing events are caused by binary lenses. Here we review the probability, current known events and discuss future prospects.

\section{Cross Sections and Light Curves}

The cross section of detecting binary systems is closely related to the caustic structures of binary and planetary systems. Depending on the binary and planetary separation and mass ratio distributions, the probability of binary and planetary lensing can be of the order of 5\%-10\% (Mao \& Paczyński 1991) or even higher (Gould \& Loeb 1992; Bolatto \& Falco 1994).

The light curves of binary events are very diverse (Mao \& Paczyński 1991 and references therein). The most dramatic ones are the caustic crossing events with sharp spikes. These can be unambiguously identified. Less dramatic ones will be more difficult to detect. The fitting of a binary light curve involves many parameters (Mao \& Di Stefano 1995). Degeneracy may occur when the light curve has large errors and sparse sampling.

\section{Candidate Events and Evidence for Blending}

The first binary event, OGLE \#7, was discovered by Udalski et al. (1994), and confirmed by Alcock et al. (1995b). It lasted for $\sim 100$ days and has two 
sharp spikes, the second being resolved (Alcock et al. 1995b). The second binary event (Alard et al. 1995), DUO 2, is much shorter ( $\approx 8$ days) but has three peaks. This candidate is likely to be a very low mass system, with the secondary mass in the range of a brown dwarf or a giant Jupiter.

Remarkably, both events show blending (Udalski et al. 1994, Alard et al. 1995), i.e., an unlensed source is contributing to the total brightness of the light curve. For OGLE \#7, the plateau between the spikes has a magnification of 2.4 in the I band, which is below the theoretical minimum of 3 , therefore blending must be present (Witt \& Mao 1995). For DUO 2, observations under good seeing revealed two components separated by $\approx 1^{\prime \prime}$, just as predicted by the model. Blending seems to be rather pervasive. This may have important implications for the estimate of optical depth.

\section{The Motion of the Images of a Binary}

The motion of the centroid of the images of a binary system yields additional information and would further constrains the binary parameters $(\S 2)$. For a star crossing a caustic, the light centroid can jump about $0.001-0.01^{\prime \prime}$. This motion can be potentially detected (see e.g. Armstrong et al. 1995).

\section{Outlook}

The discovery of two binary events provides us with confidence that such events can be identified. Such search can naturally be extended to planetary mass regimes, although the time coverage has to be once or a few times per hour. Such a project is already being pursued (Tytler 1995), with the goal of finding hundreds of Earth-like objects in a decade.

\section{References}

Alard, C., Mao, S. \& Guibert, J., 1995, A\&A, in press

Alcock, C., et al., 1993, Nature, 365, 621

Alcock, C., et al., 1995a, Phys Rev Letters, 74, 2867

Alcock, C., et al., 1995b, in preparation

Armstrong, J.T., et al., 1995, Physics Today, 48 (5), 42

Aubourg, E., et al., 1993, Nature, 365, 623

Bennett, D.P., et al., 1994, in Dark Matter, ed. S. Holt, in press

Bolatto, A. D., \& Falco, E. E., 1994, ApJ, 436, 112

Gould, A., \& Loeb, A., 1992, ApJ, 396, 104

Mao, S., \& Paczyński, B., 1991, ApJ, 374, L37

Mao, S., \& Di Stefano, R., 1995, ApJ, 440, 22

Paczyński, B., et al., 1994, AJ, 107, 2060

Stanek, K., et al., 1994, ApJ, 429, L73

Tytler, D., 1995, talk at the Extra-Solar Terrestrial Planet Detection Workshop

Udalski, A., et al., 1994, ApJL, 436, L103

Witt, H.J. \& Mao, S., 1995, ApJL, in press 